\title{
DISCONTINUOUS GALERKIN AND THE CROUZEIX-RAVIART ELEMENT: APPLICATION TO ELASTICITY
}

\author{
Peter Hansbo $^{1}$ and Mats G. Larson ${ }^{2}$
}

\begin{abstract}
We propose a discontinuous Galerkin method for linear elasticity, based on discontinuous piecewise linear approximation of the displacements. We show optimal order a priori error estimates, uniform in the incompressible limit, and thus locking is avoided. The discontinuous Galerkin method is closely related to the non-conforming Crouzeix-Raviart (CR) element, which in fact is obtained when one of the stabilizing parameters tends to infinity. In the case of the elasticity operator, for which the CR element is not stable in that it does not fulfill a discrete Korn's inequality, the discontinuous framework naturally suggests the appearance of (weakly consistent) stabilization terms. Thus, a stabilized version of the CR element, which does not lock, can be used for both compressible and (nearly) incompressible elasticity. Numerical results supporting these assertions are included. The analysis directly extends to higher order elements and three spatial dimensions.
\end{abstract}

Mathematics Subject Classification. 65N30, 74B05.

Received: July 5, 2001.

\section{INTRODUCTION}

In a discontinuous Galerkin method the approximation space typically consists of discontinuous piecewise polynomials with boundary conditions and continuity on inter-element boundaries weakly enforced through the bilinear form. For second order problems these methods appear to origin from the work of Nitsche [11], where a consistent method for weak imposement of Dirichlet conditions was introduced. Later, similar techniques to enforce continuity on inter element boundaries were introduced and analyzed, see for instance Baker [2], Wheeler [14] and Arnold [1]. Recently there has been a growing interest in discontinuous Galerkin methods for a variety of different applications, see the conference proceedings [15] for an overview of recent work.

In this paper we propose and analyze a discontinuous Galerkin method for linear elasticity, which has optimal order and does not lock in the incompressible limit. The method is related to the earlier work of Hansbo and Larson [8], but uses a different stabilization (or penalization) of discontinuities. There is a natural connection between the discontinuous Galerkin method proposed here and the classical nonconforming Crouzeix-Raviart (CR) element, see [5]. The lowest order CR element is a simple nonconforming finite element for triangular elements with nodes situated at the midpoints of the element sides, which can be used for the Poisson problem and the Stokes or Navier-Stokes problems [5,13]. Further, Brenner and Sung [3] used the CR element to construct a locking free method for the pure displacement problem of almost incompressible elasticity. However,

\footnotetext{
Keywords and phrases. Crouzeix-Raviart element, Nitsche's method, discontinuous Galerkin, incompressible elasticity.

1 Department of Applied Mechanics, Chalmers University of Technology, S-412 96 Göteborg, Sweden.

2 Department of Mathematics, Chalmers University of Technology, S-412 96 Göteborg, Sweden.
} 
for the traction problem in elasticity the CR element is known to be unstable, since it can not control the rigid body rotations, cf. Hughes [10] (Sect. 4.7). In the two dimensional case, Falk [6] obtained a stable version of the CR element by splitting the elasticity operator and projecting one part of the operator onto a macro element. In this paper, we instead obtain a stabilized method for the CR element, by simply using the CR element in the discontinuous Galerkin method. In fact, the CR element approximation is obtained as a certain stabilization parameter tends to infinity in the discontinuous Galerkin method. Thus, error estimates for the nonconforming method based on the CR element are obtained as special cases of the error estimates for the discontinuous Galerkin method. Although we present the analysis of linear elements in two spatial dimensions it directly extends to higher order elements and three spatial dimensions. For simplicity, the analysis is done for the case of homogeneous Dirichlet boundary conditions, but the analysis can be easily extended to the mixed boundary-value problem provided the necessary regularity results hold.

The paper is organized as follows: in Section 2 we introduce the equations of elasticity and the discontinuous Galerkin method, and we prove a priori error estimates; in Section 3 we introduce the stabilized method for the nonconforming CR element; and in Section 4 we present numerical examples illustrating our results.

\section{A discontinuous Galerkin method for Elasticity}

\subsection{The equations of elasticity}

We consider the equations of linear elasticity describing the displacement of an elastic body occupying a domain $\Omega$ in two spatial dimensions: find the displacement $\boldsymbol{u}=\left[u_{i}\right]_{i=1}^{2}$ and the symmetric stress tensor $\boldsymbol{\sigma}=\left[\sigma_{i j}\right]_{i, j=1}^{2}$ such that

$$
\begin{aligned}
\boldsymbol{\sigma} & =\lambda \operatorname{tr} \boldsymbol{\varepsilon}(\boldsymbol{u}) \boldsymbol{I}+2 \mu \boldsymbol{\varepsilon}(\boldsymbol{u}) & & \text { in } \Omega, \\
-\nabla \cdot \boldsymbol{\sigma} & =\boldsymbol{f} & & \text { in } \Omega, \\
\boldsymbol{u} & =\mathbf{0} & & \text { on } \partial \Omega .
\end{aligned}
$$

Here $\boldsymbol{\varepsilon}(\boldsymbol{u})=\left[\varepsilon_{i j}(\boldsymbol{u})\right]_{i, j=1}^{2}$ is the strain tensor with components

$$
\varepsilon_{i j}(\boldsymbol{u})=\frac{1}{2}\left(\frac{\partial u_{i}}{\partial x_{j}}+\frac{\partial u_{j}}{\partial x_{i}}\right),
$$

$\nabla \cdot \boldsymbol{\sigma}=\left[\sum_{j=1}^{2} \partial \sigma_{i j} / \partial x_{j}\right]_{i=1}^{2}, \boldsymbol{I}=\left[\delta_{i j}\right]_{i, j=1}^{2}$ with $\delta_{i j}=1$ if $i=j$ and $\delta_{i j}=0$ if $i \neq j, \operatorname{tr} \boldsymbol{\varepsilon}(\boldsymbol{u})=\sum_{k} \varepsilon_{k k}(\boldsymbol{u})=\nabla \cdot \boldsymbol{u}$, and $\boldsymbol{f}$ is a given load. Furthermore, $\lambda$ and $\mu$ are the Lamé constants, satisfying $0<\mu_{1}<\mu<\mu_{2}$ and $0<\lambda<\infty$. Incompressible behavior is obtained as the parameter $\lambda \rightarrow \infty$.

\subsection{Formulation of the discontinuous Galerkin method}

Consider a subdivision $\mathcal{T}=\{T\}$ of $\Omega$ of $\Omega$ into a geometrically conforming finite element mesh. with $h_{T}$ the diameter of triangle $T$ and global mesh size parameter $h=\max _{T \in \mathcal{T}} h_{T}$. For simplicity, we assume that $\mathcal{T}$ is quasi uniform. Let

$$
\boldsymbol{D F}=\left\{\boldsymbol{v} \in\left[L_{2}(\Omega)\right]^{2}:\left.\boldsymbol{v}\right|_{T} \in P^{1}(T) \text { for all } T \in \mathcal{T}\right\}
$$

be the space of piecewise linear discontinuous functions. The set of edges in the mesh is denoted by $\mathcal{E}=\{E\}$ and we split $\mathcal{E}$ into two disjoint subsets

$$
\mathcal{E}=\mathcal{E}_{\mathrm{I}} \cup \mathcal{E}_{\mathrm{E}}
$$

where $\mathcal{E}_{\mathrm{I}}$ is the set of edges in the interior of $\Omega$ and $\mathcal{E}_{\mathrm{E}}$ is the set of edges on the boundary $\partial \Omega$. Further, with each edge we associate a fixed unit normal $\boldsymbol{n}$ such that for edges on the boundary $\boldsymbol{n}$ is the exterior unit normal. 
We denote the jump of a function $\boldsymbol{v} \in \boldsymbol{D} \boldsymbol{F}$ at an edge $E$ by $[\boldsymbol{v}]=\boldsymbol{v}^{+}-\boldsymbol{v}^{-}$for $E \in \mathcal{E}_{\mathrm{I}}$ and $[\boldsymbol{v}]=\boldsymbol{v}^{+}$for $E \in \mathcal{E}_{\mathrm{E}}$, and the average $\langle\boldsymbol{v}\rangle=\left(\boldsymbol{v}^{+}+\boldsymbol{v}^{-}\right) / 2$ for $E \in \mathcal{E}_{\mathrm{I}}$ and $\langle\boldsymbol{v}\rangle=\boldsymbol{v}^{+}$for $E \in \mathcal{E}_{\mathrm{E}}$, where $\boldsymbol{v}^{ \pm}=\lim _{\epsilon \downarrow 0} \boldsymbol{v}(\boldsymbol{x} \mp \epsilon \boldsymbol{n})$ with $\boldsymbol{x} \in E$.

The discontinuous Galerkin method reads: find $\boldsymbol{U} \in \boldsymbol{D F}$ such that

$$
a(\boldsymbol{U}, \boldsymbol{v})=l(\boldsymbol{v}) \quad \text { for all } \boldsymbol{v} \in \boldsymbol{D F} .
$$

The bilinear form is defined by

$$
\begin{aligned}
a(\boldsymbol{U}, \boldsymbol{v})= & \sum_{T \in \mathcal{T}}(\boldsymbol{\sigma}(\boldsymbol{U}), \boldsymbol{\varepsilon}(\boldsymbol{v}))_{T}-\sum_{E \in \mathcal{E}}(\langle\boldsymbol{n} \cdot \boldsymbol{\sigma}(\boldsymbol{U})\rangle,[\boldsymbol{v}])_{E}+(\langle\boldsymbol{n} \cdot \boldsymbol{\sigma}(\boldsymbol{v})\rangle,[\boldsymbol{U}])_{E} \\
& +(2 \mu+\lambda) \gamma_{0} \sum_{E \in \mathcal{E}}\left(h^{-1}\left[P_{0} \boldsymbol{U}\right],\left[P_{0} \boldsymbol{v}\right]\right)_{E}+2 \mu \gamma_{1} \sum_{E \in \mathcal{E}}\left(h^{-1}[\boldsymbol{U}],[\boldsymbol{v}]\right)_{E}
\end{aligned}
$$

and the linear functional is defined by

$$
l(\boldsymbol{v})=\sum_{T \in \mathcal{T}}(\boldsymbol{f}, \boldsymbol{v})_{T}
$$

Here

$$
(\boldsymbol{\sigma}, \boldsymbol{\varepsilon})_{T}=\int_{T} \boldsymbol{\sigma}: \boldsymbol{\varepsilon}=\int_{T} \sum_{i j} \boldsymbol{\sigma}_{i j} \boldsymbol{\varepsilon}_{i j}
$$

for 2-tensors $\boldsymbol{\sigma}, \boldsymbol{\varepsilon} ;(\boldsymbol{v}, \boldsymbol{w})_{E}=\int_{E} \boldsymbol{v} \cdot \boldsymbol{w}$ for vectors $\boldsymbol{v}, \boldsymbol{w} ; P_{0}$ is the $L_{2}$-projection onto constants on each edge $E$, i.e.,

$$
\left.P_{0} \boldsymbol{v}\right|_{E}=\frac{1}{|E|} \int_{E} \boldsymbol{v}
$$

with $|E|$ the length of $E ; h$ is defined by

$$
\left.h\right|_{E}=\left(\left|T^{+}\right|+\left|T^{-}\right|\right) /(2|E|) \text { for } E=\partial T^{+} \cap \partial T^{-},
$$

with $|T|$ the area of $T$, on each edge.

Using Green's formula, we readily establish the following proposition.

Proposition 2.1. The method (4) is consistent in the sense that

$$
a(\boldsymbol{u}-\boldsymbol{U}, \boldsymbol{v})=0 \quad \text { for all } \boldsymbol{v} \in \boldsymbol{D F}
$$

and $\boldsymbol{u}$ sufficiently regular.

\subsection{A priori error estimates}

For the purpose of error analysis, we introduce the following mesh dependent energy-like norm

$$
\|\boldsymbol{v}\|^{2}=\sum_{T \in \mathcal{T}}(\boldsymbol{\sigma}(\boldsymbol{v}), \boldsymbol{\varepsilon}(\boldsymbol{v}))_{T}+\sum_{E \in \mathcal{E}}(h\langle\boldsymbol{n} \cdot \boldsymbol{\sigma}(\boldsymbol{v})\rangle,\langle\boldsymbol{n} \cdot \boldsymbol{\varepsilon}(\boldsymbol{v})\rangle)_{E}+\sum_{E \in \mathcal{E}}\left(\frac{2 \mu}{h}[\boldsymbol{v}],[\boldsymbol{v}]\right)_{E}
$$

and the edge norm

$$
\|\boldsymbol{v}\|_{\mathcal{E}}^{2}=\sum_{E \in \mathcal{E}}\|\boldsymbol{v}\|_{L_{2}(E)}^{2}
$$


In order to show that the method (4) is stable, we shall show that $a(\cdot, \cdot)$ is coercive with respect to the norm $\|\cdot\|$, given that $\gamma_{0}$ is sufficiently large and $\gamma_{1}$ is positive and bounded away from zero. We also give a bound on the piecewise constant part of the jumps at each edge.

Proposition 2.2. If $\gamma_{0}>c_{0}$, with $c_{0}$ sufficiently large and $\gamma_{1} \geq c_{1}>0$, then the following estimates hold

$$
c|\|\boldsymbol{v}\||^{2}+\alpha\left(\lambda, \gamma_{0}\right)\left\|h^{-1 / 2}\left[P_{0} \boldsymbol{v}\right]\right\|_{\mathcal{E}}^{2} \leq a(\boldsymbol{v}, \boldsymbol{v}),
$$

for all $\boldsymbol{v} \in \boldsymbol{D F}$. Here $\alpha\left(\lambda, \gamma_{0}\right)=(2 \mu+3 \lambda)\left(\gamma_{0}-c_{0}\right)$ and the constant $c$ is independent of $h, \gamma_{0}, \gamma_{1}, \mu$, and $\lambda$.

Proof. We first note that the following inverse estimate holds

$$
\sum_{E \in \mathcal{E}}\left(h^{1 / 2}\langle\boldsymbol{n} \cdot \boldsymbol{\sigma}(\boldsymbol{v})\rangle,\langle\boldsymbol{n} \cdot \boldsymbol{\varepsilon}(\boldsymbol{v})\rangle\right)_{E} \leq C_{\mathrm{i}} \sum_{T \in \mathcal{T}}(\boldsymbol{\sigma}(\boldsymbol{v}), \boldsymbol{\varepsilon}(\boldsymbol{v}))_{T} .
$$

This inequality is proved by scaling and finite dimensionality, cf. Arnold [1]. We also note that since (1) can be inverted to yield

$$
\boldsymbol{\varepsilon}=\frac{1}{2 \mu}\left(\boldsymbol{\sigma}-\frac{\lambda}{3 \lambda+2 \mu} \operatorname{tr} \boldsymbol{\sigma} \boldsymbol{I}\right)=\frac{1}{2 \mu} \boldsymbol{\sigma}^{D}+\frac{1}{9 \lambda+6 \mu} \operatorname{tr} \boldsymbol{\sigma} \boldsymbol{I}
$$

where $\boldsymbol{\sigma}^{D}=\boldsymbol{\sigma}-\operatorname{tr} \boldsymbol{\sigma} \boldsymbol{I} / 3$, we have that

$$
\boldsymbol{\sigma}: \boldsymbol{\varepsilon}=\frac{1}{2 \mu} \boldsymbol{\sigma}^{D}: \boldsymbol{\sigma}^{D}+\frac{1}{9 \lambda+6 \mu}(\operatorname{tr} \boldsymbol{\sigma})^{2} \quad \text { and } \quad \boldsymbol{\sigma}: \boldsymbol{\sigma}=\boldsymbol{\sigma}^{D}: \boldsymbol{\sigma}^{D}+\frac{1}{3}(\operatorname{tr} \boldsymbol{\sigma})^{2}
$$

so that $\boldsymbol{\sigma}: \boldsymbol{\sigma} \leq(2 \mu+3 \lambda) \boldsymbol{\sigma}: \boldsymbol{\varepsilon}$. Thus

$$
\frac{1}{2 \mu+3 \lambda}\|\boldsymbol{n} \cdot \boldsymbol{\sigma}(\boldsymbol{v})\|_{L_{2}(E)}^{2} \leq(\boldsymbol{n} \cdot \boldsymbol{\sigma}(\boldsymbol{v}), \boldsymbol{n} \cdot \boldsymbol{\varepsilon}(\boldsymbol{v}))_{E} .
$$

Further, using that $\langle\boldsymbol{n} \cdot \boldsymbol{\sigma}(\boldsymbol{v})\rangle$ is constant, we have, for each $E \in \mathcal{E}$ and for $\delta>0$, that

$$
\begin{aligned}
2(\langle\boldsymbol{n} \cdot \boldsymbol{\sigma}(\boldsymbol{v})\rangle,[\boldsymbol{v}])_{E} & =2\left(\langle\boldsymbol{n} \cdot \boldsymbol{\sigma}(\boldsymbol{v})\rangle,\left[P_{0} \boldsymbol{v}\right]\right)_{E} \\
& \leq \frac{2 \delta}{2 \mu+3 \lambda}\left\|h^{1 / 2}\langle\boldsymbol{n} \cdot \boldsymbol{\sigma}(\boldsymbol{v})\rangle\right\|_{L_{2}(E)}^{2}+\frac{2 \mu+3 \lambda}{\delta / 2}\left\|h^{-1 / 2}\left[P_{0} \boldsymbol{v}\right]\right\|_{L_{2}(E)}^{2} \\
& \leq 2 \delta\left(h^{1 / 2}\langle\boldsymbol{n} \cdot \boldsymbol{\sigma}(\boldsymbol{v})\rangle,\langle\boldsymbol{n} \cdot \boldsymbol{\varepsilon}(\boldsymbol{v})\rangle\right)_{E}+(2 \mu+3 \lambda)(\delta / 2)^{-1}\left\|h^{-1 / 2}\left[P_{0} \boldsymbol{v}\right]\right\|_{L_{2}(E)}^{2}
\end{aligned}
$$

where we used the Cauchy-Schwarz inequality followed by the arithmetic-geometric mean inequality. Using these estimates we obtain

$$
\begin{aligned}
a(\boldsymbol{v}, \boldsymbol{v}) \geq & \left(1-C_{\mathrm{i}} \delta\right) \sum_{T \in \mathcal{T}}(\boldsymbol{\sigma}(\boldsymbol{v}), \boldsymbol{\varepsilon}(\boldsymbol{v}))_{T}+\delta \sum_{E \in \mathcal{E}}\left(h^{1 / 2}\langle\boldsymbol{n} \cdot \boldsymbol{\sigma}(\boldsymbol{v})\rangle,\langle\boldsymbol{n} \cdot \boldsymbol{\varepsilon}(\boldsymbol{v})\rangle\right)_{E} \\
& +(2 \mu+3 \lambda)\left(\gamma_{0}-2 / \delta\right)\left\|h^{-1 / 2}\left[P_{0} \boldsymbol{v}\right]\right\|_{\mathcal{E}}^{2}+2 \mu \gamma_{1}\left\|h^{-1 / 2}[\boldsymbol{v}]\right\|_{\mathcal{E}}^{2} \\
\geq & c\|\boldsymbol{v}\|^{2}+(2 \mu+3 \lambda)\left(\gamma_{0}-c_{0}\right)\left\|h^{-1 / 2}\left[P_{0} \boldsymbol{v}\right]\right\|_{\mathcal{E}}^{2}
\end{aligned}
$$

where we chose $c_{0}=2 \delta^{-1}=4 C_{\mathrm{i}}$ in the last inequality.

For the proof of our a priori error estimate we introduce the interpolation operator $\boldsymbol{\pi}:\left[H^{2}(\Omega)\right]^{2} \rightarrow \boldsymbol{D F}$ introduced by Crouzeix and Raviart in [5], and constructed as follows. At the midpoint $\boldsymbol{x}_{m}$ of the edge $E$, let

$$
\boldsymbol{\pi} \boldsymbol{u}\left(\boldsymbol{x}_{m}\right):=\frac{1}{|E|} \int_{E} \boldsymbol{u}
$$


from which it follows, by application of Gauss' theorem, cf. [5], that

$$
\left.\nabla \cdot(\boldsymbol{\pi} \boldsymbol{u})\right|_{T}=\frac{1}{|T|} \int_{T} \nabla \cdot \boldsymbol{u} .
$$

For this interpolant, we have the following basic estimates.

Lemma 2.3. The following estimates hold

$$
\begin{gathered}
\|\boldsymbol{u}-\boldsymbol{\pi} \boldsymbol{u}\|_{L_{2}(T)}+h_{T}\|\boldsymbol{u}-\boldsymbol{\pi} \boldsymbol{u}\|_{H^{1}(T)} \leq C h_{T}^{2}\|\boldsymbol{u}\|_{H^{2}(T)}, \\
\|\nabla \cdot(\boldsymbol{u}-\boldsymbol{\pi} \boldsymbol{u})\|_{L_{2}(T)}+h_{T}\|\nabla \cdot(\boldsymbol{u}-\boldsymbol{\pi} \boldsymbol{u})\|_{H^{1}(T)} \leq C h_{T}\|\nabla \cdot \boldsymbol{u}\|_{H^{1}(T)} .
\end{gathered}
$$

Proof. The proof of (15) can be found in [5], and (16) follows from (14).

For our a priori error estimate we shall need the following lemma.

Lemma 2.4. The following interpolation estimate holds

$$
\|\boldsymbol{u}-\boldsymbol{\pi} \boldsymbol{u}\| \mid \leq C h\left((2 \mu)^{1 / 2}\|\boldsymbol{u}\|_{H^{2}(\Omega)}+\lambda^{1 / 2}\|\nabla \cdot \boldsymbol{u}\|_{H^{1}(\Omega)}\right) .
$$

Proof. We first recall the trace inequality

$$
h_{T}^{-1 / 2}\|\boldsymbol{w}\|_{L_{2}(\partial T)} \leq C\left(h_{T}^{-1}\|\boldsymbol{w}\|_{L_{2}(T)}+\|\boldsymbol{w}\|_{H^{1}(T)}\right) \quad \forall \boldsymbol{w} \in H^{1}(T),
$$

see, e.g., Thomée [4]. For the inequality (17), the interior part is estimated directly using Lemma 2.3. For the contribution from the jumps, we have using the triangle inequality

$$
\left\|h^{-1 / 2}[\boldsymbol{u}-\boldsymbol{\pi} \boldsymbol{u}]\right\|_{\mathcal{E}} \leq \sum_{T \in \mathcal{T}}\left\|h^{-1 / 2}(\boldsymbol{u}-\boldsymbol{\pi} \boldsymbol{u})\right\|_{\partial T}
$$

Next, using the trace inequality and Lemma 2.3, we get

$$
\left\|h^{-1 / 2}(\boldsymbol{u}-\boldsymbol{\pi} \boldsymbol{u})\right\|_{\partial T} \leq C\left(h_{T}^{-1}\|\boldsymbol{u}-\boldsymbol{\pi} \boldsymbol{u}\|_{L_{2}(T)}+\|\boldsymbol{u}-\boldsymbol{\pi} \boldsymbol{u}\|_{H^{1}(T)}\right) \leq C h_{T}\|\boldsymbol{u}\|_{H^{2}(T)} .
$$

Furthermore,

$$
(\boldsymbol{n} \cdot \boldsymbol{\sigma}(\boldsymbol{v}), \boldsymbol{n} \cdot \boldsymbol{\varepsilon}(\boldsymbol{v}))_{\partial T}=2 \mu(\boldsymbol{n} \cdot \boldsymbol{\varepsilon}(\boldsymbol{v}), \boldsymbol{n} \cdot \boldsymbol{\varepsilon}(\boldsymbol{v}))_{\partial T}+\lambda(\nabla \cdot \boldsymbol{v}, \nabla \cdot \boldsymbol{v})_{\partial T},
$$

so we can use this split for the mean traction contributions, apply the triangle inequality, and estimate termwise:

$$
\left\|h^{1 / 2} \nabla \cdot(\boldsymbol{u}-\boldsymbol{\pi} \boldsymbol{u})\right\|_{L_{2}(\partial T)} \leq C\left(\|\nabla \cdot(\boldsymbol{u}-\boldsymbol{\pi} \boldsymbol{u})\|_{L_{2}(T)}+h_{T}\|\nabla \cdot(\boldsymbol{u}-\boldsymbol{\pi} \boldsymbol{u})\|_{H^{1}(T)}\right) \leq C h_{T}\|\nabla \cdot \boldsymbol{u}\|_{H^{1}(T)},
$$

and

$$
\begin{aligned}
\left\|h^{1 / 2} \boldsymbol{n} \cdot \boldsymbol{\varepsilon}(\boldsymbol{u}-\boldsymbol{\pi} \boldsymbol{u})\right\|_{L_{2}(\partial T)} & \leq C\left(\|\boldsymbol{\varepsilon}(\boldsymbol{u}-\boldsymbol{\pi} \boldsymbol{u})\|_{L_{2}(T)}+h_{T}\|\boldsymbol{\varepsilon}(\boldsymbol{u}-\boldsymbol{\pi} \boldsymbol{u})\|_{H^{1}(T)}\right) \\
& \leq C\left(\|\boldsymbol{u}-\boldsymbol{\pi} \boldsymbol{u}\|_{H^{1}(T)}+h_{T}\|\boldsymbol{u}\|_{H^{2}(T)}\right) \\
& \leq C h_{T}\|\boldsymbol{u}\|_{H^{2}(T)},
\end{aligned}
$$

and the lemma follows after summation. 
We are now ready to show our main result.

Theorem 2.5. With $\boldsymbol{u}$ the solution of (1)-(3) and $\boldsymbol{U}$ the solution of (4), we have under the assumptions of Proposition 2.2,

$$
\|\boldsymbol{u}-\boldsymbol{U}\|+\alpha\left(\lambda, \gamma_{0}\right)^{1 / 2}\left\|h^{-1 / 2}\left[P_{0} \boldsymbol{U}\right]\right\|_{\mathcal{E}} \leq C h\left((2 \mu)^{1 / 2}\|\boldsymbol{u}\|_{H^{2}(\Omega)}+\lambda^{1 / 2}\|\nabla \cdot \boldsymbol{u}\|_{H^{1}(\Omega)}\right),
$$

where $\alpha(\cdot, \cdot)$ is defined in Proposition 2.2 and $C$ is a constant independent of $h, \gamma_{0}, \gamma_{1}, \mu$, and $\lambda$.

Proof. By the triangle inequality, we have that

$$
\|\boldsymbol{u}-\boldsymbol{U}\||\leq\|\boldsymbol{U}-\boldsymbol{\pi} \boldsymbol{u}\||+\|\boldsymbol{u}-\boldsymbol{\pi} \boldsymbol{u}\| \mid
$$

and from coercivity, Proposition 2.2, and consistency, Proposition 2.1, it follows that

$$
c \mid\|\boldsymbol{U}-\boldsymbol{\pi} \boldsymbol{u}\|\left\|^{2}+\alpha\left(\lambda, \gamma_{0}\right)\right\| h^{-1 / 2}\left[P_{0} \boldsymbol{U}\right] \|_{\mathcal{E}}^{2} \leq a(\boldsymbol{U}-\boldsymbol{\pi} \boldsymbol{u}, \boldsymbol{U}-\boldsymbol{\pi} \boldsymbol{u})=a(\boldsymbol{u}-\boldsymbol{\pi} \boldsymbol{u}, \boldsymbol{U}-\boldsymbol{\pi} \boldsymbol{u})
$$

To estimate the right-hand side $a(\boldsymbol{u}-\boldsymbol{\pi} \boldsymbol{u}, \boldsymbol{U}-\boldsymbol{\pi} \boldsymbol{u})$, we use the continuity of the bilinear form to get

$$
a(\boldsymbol{u}-\boldsymbol{\pi} \boldsymbol{u}, \boldsymbol{U}-\boldsymbol{\pi} \boldsymbol{u}) \leq C|\|\boldsymbol{U}-\boldsymbol{\pi} \boldsymbol{u}\||\|\boldsymbol{u}-\boldsymbol{\pi} \boldsymbol{u}\| \mid .
$$

Combining (19) and (20), we find that

$$
\|\boldsymbol{U}-\boldsymbol{\pi} \boldsymbol{u}|\|\leq C|\|\boldsymbol{u}-\boldsymbol{\pi} \boldsymbol{u}\||
$$

and thus

$$
a(\boldsymbol{u}-\boldsymbol{\pi} \boldsymbol{u}, \boldsymbol{U}-\boldsymbol{\pi} \boldsymbol{u}) \leq\left. C\|\boldsymbol{u}-\boldsymbol{\pi} \boldsymbol{u}\|\right|^{2}
$$

Finally, using the interpolation estimates of Lemma 2.4, the bound follows.

Combining the error estimate in Theorem 2.5 with the elliptic regularity estimate

$$
\|\boldsymbol{u}\|_{H^{2}(\Omega)}+\lambda\|\nabla \cdot \boldsymbol{u}\|_{H^{1}(\Omega)} \leq C\|\boldsymbol{f}\|_{L_{2}(\Omega)},
$$

which holds for instance in the case of a convex polygonal domain $\Omega$, cf. Brenner and Sung [3], we obtain the following estimate in terms of data, which shows that the method does not lock as $\lambda \rightarrow \infty$.

Corollary 2.6. There is a constant $C$, independent of $h, \gamma_{0}, \gamma_{1}, \mu$, and $\lambda$ such that

$$
\|\boldsymbol{u}-\boldsymbol{U}\| \mid+\alpha\left(\lambda, \gamma_{0}\right)^{1 / 2}\left\|h^{-1 / 2}\left[P_{0} \boldsymbol{U}\right]\right\|_{\mathcal{E}} \leq C h\|\boldsymbol{f}\|_{L_{2}(\Omega)} .
$$

In case (22) holds, we also have the following $L_{2}$-norm estimate:

Corollary 2.7. With $\boldsymbol{u}$ the solution of (1)-(3) and $\boldsymbol{U}$ the solution of (4), we have

$$
\|\boldsymbol{u}-\boldsymbol{U}\|_{L_{2}(\Omega)} \leq C h^{2}\|\boldsymbol{f}\|_{L_{2}(\Omega)} .
$$

Proof. For a given $\boldsymbol{w} \in L_{2}(\Omega)$, define $\boldsymbol{z}$ as the solution to the problem

$$
-\nabla \cdot(\lambda \nabla \cdot \boldsymbol{z} \boldsymbol{I}+2 \mu \boldsymbol{\varepsilon}(\boldsymbol{z}))=\boldsymbol{w} \text { in } \Omega, \quad \boldsymbol{z}=0 \text { on } \partial \Omega,
$$


and $\boldsymbol{Z}$ as the corresponding numerical solution using (4). Setting $\boldsymbol{e}=\boldsymbol{u}-\boldsymbol{U}$, we then have, using integration by parts and Proposition 2.1, that

$$
\sum_{T \in \mathcal{T}}(\boldsymbol{e}, \boldsymbol{w})_{T}=a(\boldsymbol{e}, \boldsymbol{z})=a(\boldsymbol{e}, \boldsymbol{z}-\boldsymbol{Z})
$$

so that, by Corollary 2.6,

$$
\left|\sum_{T \in \mathcal{T}}(\boldsymbol{e}, \boldsymbol{w})_{T}\right| \leq C\left|\|\boldsymbol{e}\|\|\| \boldsymbol{z}-\boldsymbol{Z}\|\mid \leq C h\| \boldsymbol{w}\left\|_{L_{2}(\Omega)}\right\| \boldsymbol{e}\|\| .\right.
$$

Using again Corollary 2.6 and setting $\boldsymbol{w}=\boldsymbol{e}$ concludes the proof.

\section{A nonconforming Galerkin method based on the Crouzeix-Raviart element}

Restricting the discontinuous Galerkin method to the space of Crouzeix-Raviart functions

$$
\boldsymbol{C R}=\left\{\boldsymbol{v} \in \boldsymbol{D F}:\left[P_{0} \boldsymbol{v}\right]=0, \text { for all } E \in \mathcal{E}\right\},
$$

we obtain the following simplified scheme. Find $\boldsymbol{U} \in \boldsymbol{C} \boldsymbol{R}$ such that

$$
a(\boldsymbol{U}, \boldsymbol{v})=l(\boldsymbol{v}) \text { for all } \boldsymbol{v} \in \boldsymbol{C R},
$$

where

$$
a(\boldsymbol{U}, \boldsymbol{v})=\sum_{T \in \mathcal{T}}(\boldsymbol{\sigma}(\boldsymbol{U}), \boldsymbol{\varepsilon}(\boldsymbol{v}))+2 \mu \gamma_{1} \sum_{E \in \mathcal{E}}\left(h^{-1}[\boldsymbol{U}] \cdot[\boldsymbol{v}]\right)_{E}
$$

and

$$
l(\boldsymbol{v})=\sum_{T \in \mathcal{T}}(\boldsymbol{f}, \boldsymbol{v})_{T}
$$

Using the theory developed in the previous section to this method we obtain the following theorem.

Theorem 3.1. The discontinuous Galerkin method with the CR-element (25) is the limit of the discontinuous Galerkin method (4) as $\gamma_{0} \rightarrow \infty$. If $\gamma_{1} \geq c_{1}>0$, then (25) has a unique solution $\boldsymbol{U}$ and the following error estimates holds

$$
\|\boldsymbol{u}-\boldsymbol{U}\| \leq C_{1} h\|\boldsymbol{f}\|_{L_{2}(\Omega)},
$$

and

$$
\|\boldsymbol{u}-\boldsymbol{U}\|_{L_{2}(\Omega)} \leq C_{2} h^{2}\|\boldsymbol{f}\|_{L_{2}(\Omega)},
$$

with $C_{1}, C_{2}$ independent of $\mu, \lambda$ and $h$.

Proof. The fact that the solution to the discontinuous Galerkin method (4) tends to (25) as $\gamma_{0} \rightarrow \infty$ implies that the error estimates follow directly from Corollaries 2.6 and 2.7 since the constants are independent of $\gamma_{0}$.

Remark 3.2. Our analysis extends directly to the case of higher order polynomials. For odd order of polynomials we get the CR-family of elements, while for even the situation is not so simple, due to the fact that the values of a polynomial at the Gauss points on the edges of a triangle is dependent in that case. For polynomials of order two we refer to Fortin and Soulie [7], where it is shown that one obtains the usual continuous quadratic polynomials together with a nonconforming bubble on each element. 


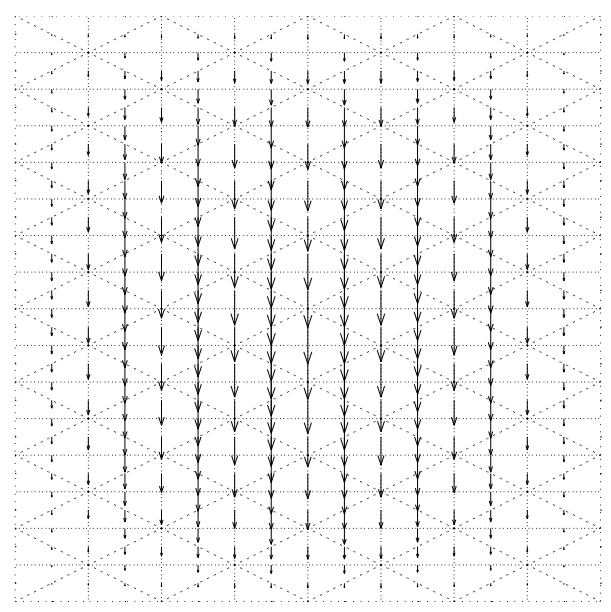

FiguRE 1. Exact solution.
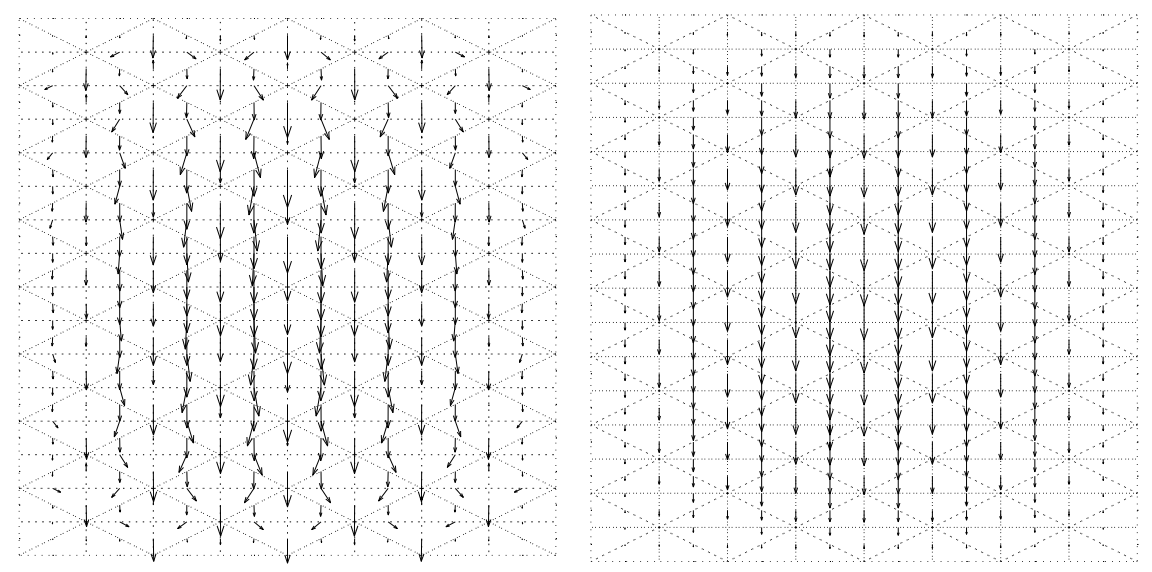

FIgURE 2. Unstable solution corresponding to choosing $\gamma_{1} \ll 1$, and stable solution for $\gamma_{1}=1$.

Remark 3.3. On rectangular elements a similar approach can be used for the element proposed by Rannacher and Turek in [12], see Hansbo and Larson [9].

\section{EXAMPLES}

\subsection{Convergence in a smooth case}

We consider the unit square $(0,1) \times(0,1)$ with $\boldsymbol{u}=0$ on the boundary and with

$$
\boldsymbol{f}=\left((\lambda+\mu)\left(1-2 x_{1}\right)\left(1-2 x_{2}\right),-2 \mu x_{2}\left(1-x_{2}\right)-2(\lambda+2 \mu) x_{1}\left(1-x_{1}\right)\right)
$$

corresponding to the exact solution

$$
\boldsymbol{u}=\left(0,-x_{1} x_{2}\left(1-x_{1}\right)\left(1-x_{2}\right)\right)
$$




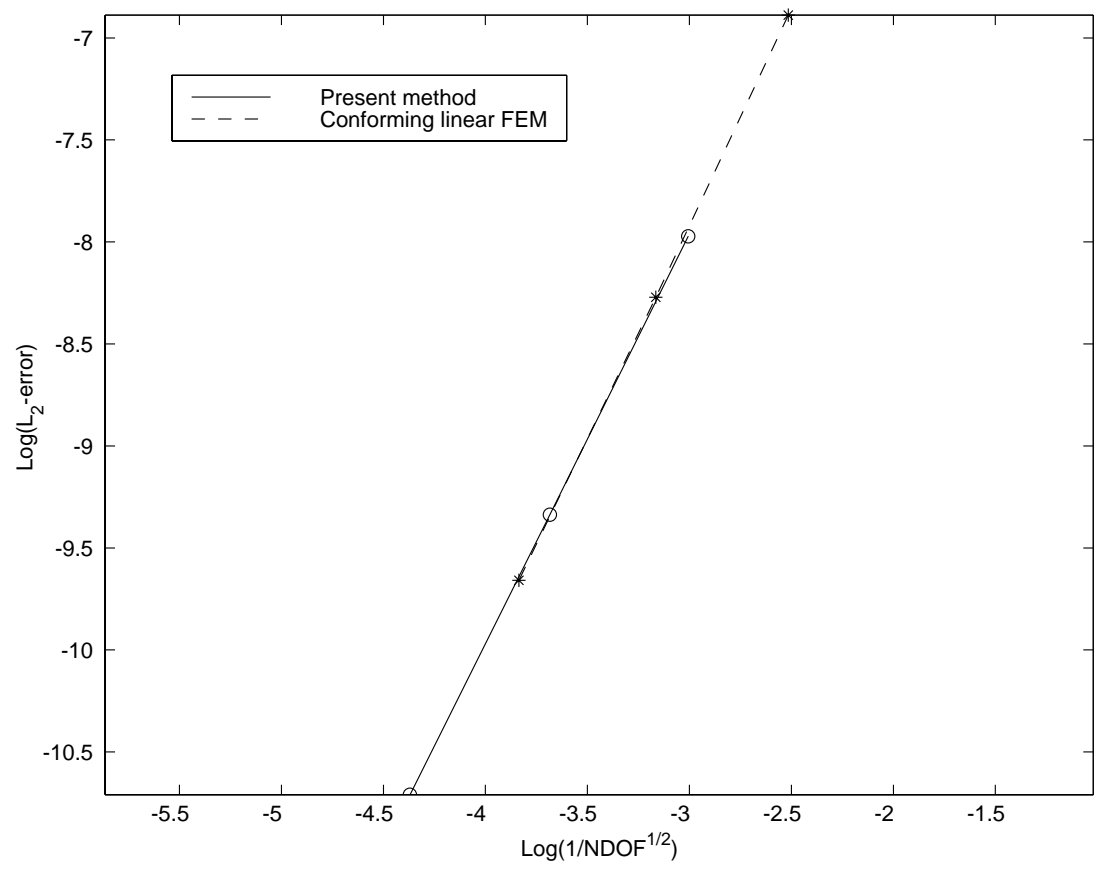

FiguRE 3. Second order convergence in the $L_{2}(\Omega)$-norm. The error relative to the number of degrees of freedom is comparable to a conforming finite element method.

The material data were chosen as $\mathrm{E}=1000$ and $\nu=1 / 10$, where

$$
\lambda=\frac{\nu \mathrm{E}}{(1+\nu)(1-2 \nu)} \text { and } \mu=\frac{\mathrm{E}}{2(1+\nu)} .
$$

A mesh with the exact solution plotted in the nodes is given in Figure 1.

In Figure 2 we show the stability problem of the CR element corresponding to choosing $\gamma_{1}$ too small, and the stabilizing effect of the boundary jumps with an adequate choice of $\gamma_{1}$.

In Figure 3 we show the convergence of the CR method (with $\gamma_{1}=0.5$ ), and second order convergence is attained, in accordance with Corollary 2.7. We also give the convergence rate of the corresponding conforming linear finite element method. It is noticeable that the error relative to the number of unknowns is almost identical for the nonconforming and conforming method.

\subsection{Locking}

On the unit square $(0,1) \times(0,1)$, we prescribe the boundary data as follows. At $x_{1}=0, x_{1}=1, x_{2}=0$ we set $\boldsymbol{u}=0$, while at $x_{2}=1$, for $0<x_{1}<1$, we set $\boldsymbol{u}=(1,0)$ to obtain the closed cavity flow problem. In Figures 4 and 5 we compare the linear conforming finite element method with the stabilized CR approximation $\left(\gamma_{1}=0.5\right)$ for $\nu \rightarrow 1 / 2$. The CR element does not lock, while the conforming method, for $\nu=0.4999$, shows clear signs of locking. Note that, due to lack of regularity, this problem is not covered by our a priori error estimate and only serves to show that locking does not occur. 

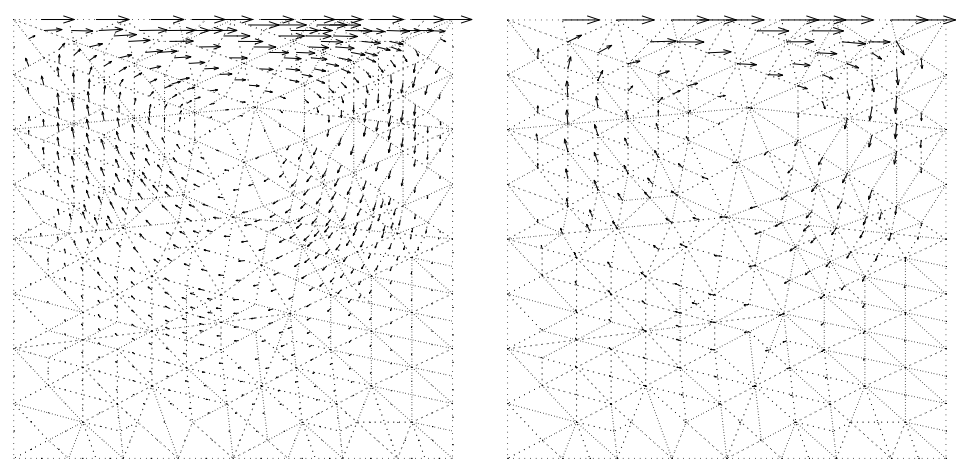

FiguRE 4. Nonconforming and conforming solutions for $\nu=0.49$.
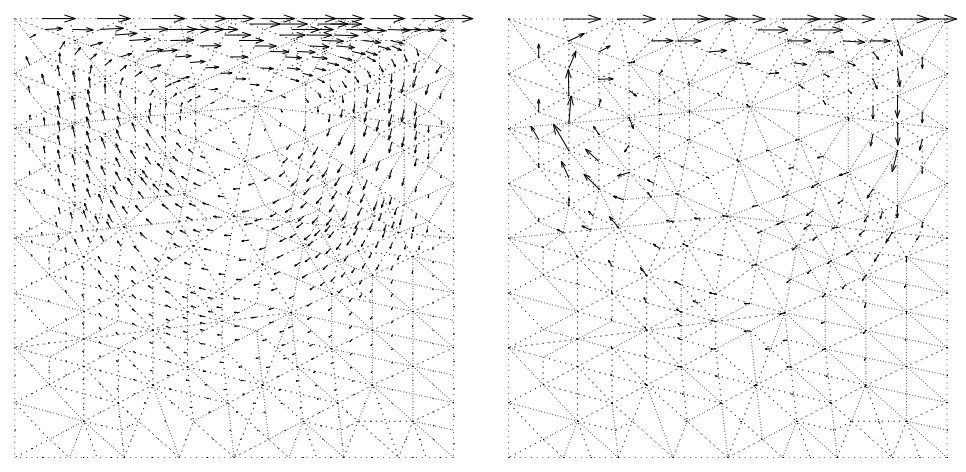

FiguRE 5. Nonconforming and (locked) conforming solutions for $\nu=0.4999$.

\section{REFERENCES}

[1] D.N. Arnold, An interior penalty finite element method with discontinuous elements. SIAM J. Numer. Anal. 19 (1982) $742-760$.

[2] G.A. Baker, Finite element methods for elliptic equations using nonconforming elements. Math. Comp. 31 (1977) 45-59.

[3] S.C. Brenner and L. Sung, Linear finite element methods for planar linear elasticity. Math. Comp. 59 (1992) 321-338.

[4] V. Thomée, Galerkin Finite Element Methods for Parabolic Problems. Springer (1997).

[5] M. Crouzeix and P.-A. Raviart, Conforming and nonconforming finite element methods for solving the stationary Stokes equations. RAIRO Sér. Rouge 7 (1973) 33-75.

[6] R.S. Falk, Nonconforming finite element methods for the equations of linear elasticity. Math. Comp. 57 (1991) 529-550.

[7] M. Fortin and M. Soulie, A nonconforming piecewise quadratic finite element on triangles. Internat. J. Numer. Methods Engrg. 19 (1983) 505-520.

[8] P. Hansbo and M.G. Larson, Discontinuous Galerkin methods for incompressible and nearly incompressible elasticity by Nitsche's method. Comput. Methods Appl. Mech. Engrg. 191 (2002) 1895-1908.

[9] P. Hansbo and M.G. Larson, A simple nonconforming bilinear element for the elasticity problem. Trends in Computational Structural Mechanics, W.A. Wall et al. Eds., CIMNE (2001) 317-327.

[10] T.J.R. Hughes, The Finite Element Method: Linear Static and Dynamic Finite Element Analysis. Prentice-Hall, New Jersey (1987).

[11] J. Nitsche, Über ein Variationsprinzip zur Lösung von Dirichlet-Problemen bei Verwendung von Teilräumen, die keinen Randbedingungen unterworfen sind. Abh. Math. Sem. Univ. Hamburg 36 (1971) 9-15.

[12] R. Rannacher and S. Turek, A simple nonconforming quadrilateral Stokes element. Numer. Methods Partial Differential Equations 8 (1992) 97-111.

[13] F. Thomasset, Implementation of Finite Element Methods for Navier-Stokes Equations. Springer-Verlag, New York (1981).

[14] M.F. Wheeler, An elliptic collocation-finite element method with interior penalties. SIAM J. Numer. Anal. 15 (1978) 152-161.

[15] B. Cockburn, K.E. Karniadakis and C.-W. Shu Eds., Discontinuous Galerkin Methods: Theory, Computation, and Applications. Lecture Notes Comput. Sci. Eng., Springer Verlag (1999). 